Abanico Veterinario. Enero-Diciembre 2021; 11:1-14. http://dx.doi.org/10.21929/abavet2021.12 Artículo Original. Recibido: 20/09/2020. Aceptado: 05/02/2021. Publicado: 27/02/2021. Clave:2020-80.

\title{
Criopreservación espermática de Ambystoma mexicanum (Shaw \& Nodder, 1798)
}

Ambystoma mexicanum sperm cryopreservation (Shaw \& Nodder, 1798)

\section{Rivera-Pacheco Juan ${ }^{11 D}$, Herrera-Barragán José ${ }^{21 D *}$, León-Galván Miguel ${ }^{3 I D}$, Ocampo-Cervantes José ${ }^{4 I D}$, Pérez-Rivero Juan ${ }^{2 I D}$, Gual-Sill Fernando ${ }^{2 I D}$}

${ }^{1}$ Maestría en Biología de la reproducción animal; Universidad Autónoma Metropolitana-Iztapalapa. San Rafael Atlixco 186, Iztapalapa CDMX, CP, 09340. ²Departamento de Producción Agrícola y Animal; Universidad Autónoma Metropolitana-Xochimilco (UAM-X), Calzada del hueso 1100, Coyoacán CDMX, CP. 04960. 3Departamento de Biología., Universidad Autónoma Metropolitana-Iztapalapa. San Rafael Atlixco 186, Iztapalapa CDMX, CP, 09340. ${ }^{4}$ Centro de Investigaciones Biológicas y Acuícolas de Cuemanco (CIBAC / UAM-X), Rinconada Cuemanco S/N, Xochimilco, CDMX; CP 16035. *Autor para correspondencia: José Antonio Herrera Barragán, Departamento de Producción Agrícola y Animal. Edificio W, piso 3. Universidad Autónoma Metropolitana-Xochimilco Calzada del hueso 1100, Coyoacán CDMX, CP. 04960. Correo electrónico: jherrerab@correo.xoc.uam.mx, jcripbiologia@gmail.com, leon@xanum.uam.mx, jocampo@correo.xoc.uam.mx, jjperez1_1999@yahoo.com, fguals@correo.xoc.uam.mx

\section{Resumen}

El Ambystoma mexicanum se encuentra en peligro de extinción en vida libre, debido a efectos antropogénicos; la criopreservación espermática para su reproducción en cautiverio, puede ayudar a su conservación ex situ. El objetivo de esta investigación fue identificar la viabilidad en fresco y post descongelación de espermatozoides provenientes de diferentes espermatóforos. Durante la temporada reproductiva se indujo en nueve ejemplares, la liberación de espermatóforos mediante la reducción de la temperatura del agua. La concentración promedio por espermatóforo fue de $2.6 \pm 0.6$ X104 espermatozoides. Se determinó en espermatozoides en fresco y post descongelación, una reducción del $30 \%$ de espermatozoides vivos y un incremento de $15 \%$ de morfología anormal. Con las lectinas WGA y PNA, unidas a FITC, se determinaron dos patrones de fluorescencia distintos con cada una, lo cual evidencio la presencia y distribución membranal de $\mathrm{N}$-acetil glucosamina, ácido siálico y manosa respectivamente. Los porcentajes de espermatozoides con cada patrón de fluorescencia mostraron diferencias asociadas al número de espermatóforos de cada liberación. Se determinaron diferencias en la viabilidad de espermatozoides obtenidos de liberaciones con diferente número de espermatóforos. El protocolo para la obtención y criopreservación espermática de $A$ mexicanum, fue eficiente como herramienta para utilizar semen criopreservado para su reproducción ex situ.

Palabras clave: Anfibio, conservación, espermatóforo, urodelo.

\begin{abstract}
Ambystoma mexicanum is in danger of extinction in free-living, due to anthropogenic actions; sperm cryopreservation for captive breeding can help in its ex-situ conservation. This research aimed to identify the viability of fresh and post-thawing sperm from different spermatophores. During the breeding season, the spermatophores releasing was induced in nine specimens by reducing water temperature. The mean concentration per spermatophorous was $2.6 \pm 0.6 \times 104$ sperm. A reduction of $30 \%$ of living sperms and an increase of $15 \%$ of abnormal morphology were determined in fresh and post-thawing sperm. With the WGA and PNA lectins bounded to FITC, two different fluorescence patterns were determined in each one, that showed the membrane presence and distribution of $\mathrm{N}$-acetyl glucosamine, sialic acid, and mannose respectively. Sperm percentages in each fluorescence pattern showed differences associated with the number of spermatophores in each release. Differences in sperm viability from releases with different
\end{abstract}


numbers of spermatophores were determined. The sperm collection and cryopreservation protocol of $A$ mexicanum were efficient as tools for using cryopreserved semen for ex situ reproduction.

Keywords: Amphibian, conservation, spermatophorous, urodele.

\section{INTRODUCCIÓN}

La disminución en las poblaciones de anfibios los han llevado en casos severos a la extinción, las principales causas son la contaminación o modificación de su hábitat, la introducción de especies exóticas invasoras y enfermedades (Catenazzi, 2015; Jiménez et al., 2017; Tietje y Rödel, 2018). Actualmente, La Unión Internacional para la Conservación de la Naturaleza (IUCN, 2020), indican que más del 85\% de las especies de anfibios de México se encuentran en alguna categoría de riesgo, debido a esta situación, la mayoría de las especies del género Ambystoma en vida silvestre, actualmente se ha optado por reproducirlos en condiciones de laboratorio (Mendoza, 2012; Khattak et al., 2014; Jiménez et al., 2017). De manera específica el Ambystoma mexicanum, está incluido en la NOM-059 - SEMARNAT- 2010 como especie en peligro de extinción (NOM-059-ECOL, 2010).

La reproducción ex situ utilizando semen criopreservado, es una herramienta de reproducción asistida en cautiverio que puede contribuir a la conservación del Ambystoma mexicanum; además de contribuir a incrementar su variabilidad genética, debido a que en la mayoría de los casos la amenaza se encuentra en su propio hábitat (Clulow et al., 2014; Jiménez et al., 2017). Sin embargo, antes de implementar un protocolo de criopreservación espermática, es necesario conocer la biología reproductiva y las características espermáticas de la especie, para lograr un mayor éxito (Chester, 2013; Silla y Byrne, 2019).

Actualmente, las técnicas de obtención de gametos (espermatozoides y óvulos) que se realizan en anfibios son altamente invasivas, pues la mayoría de los procesos requieren del sacrificio del ejemplar para la extracción de los testículos o conductos eferentes y proceder a macerarlos (Chester, 2013; Shishova et al., 2011). La mayoría de los protocolos de criopreservación utilizados en espermatozoides de diferentes especies, de anfibios, han sido extrapolados de los reportados en de peces (Comizzoli et al., 2012); mostrando resultados variables entre cada uno. Chester (2013) criopreservó espermatóforos completos de $A$ mexicanum utilizando sucrosa como principal crioprotector, reportando $84 \%$ de espermatozoides vivos después de ser descongelados; sin embargo, no reporta el parámetro de espermatozoides vivos antes de su congelación. Se sabe que la manipulación in vitro de los espermatozoides ocasiona alteraciones en su membrana plasmática, en la cual se ha descrito la presencia de carbohidratos membranales, los cuales tienen una función en el reconocimiento entre gametos para lograr la fertilización (Peláez et al., 2011). 
Debido a que es limitada la base de datos sobre estudios morfológicos y de criopreservación en espermatozoides de anfibios, en específico del orden Urodelo (Browne y Chester, 2011; Chester, 2013; Sunny et al., 2014).

El objetivo de esta investigación fue identificar los parámetros de evaluación básica y características membranales en espermatozoides provenientes de diferentes ejemplares y espermatóforos de cada uno, para evaluar un protocolo de criopreservación que permita mantener su capacidad fertilizante post descongelación.

\section{MATERIAL Y MÉTODOS}

\section{Cuidado y bienestar}

El manejo de los ajolotes se llevó a cabo en las instalaciones del Centro de Investigaciones Biológicas y Acuícolas de Cuemanco (CIBAC-UAMX), con apego al Plan de Manejo, autorizado por la Secretaría de Medio Ambiente y Recursos Naturales (SEMARNAT) a la Unidad de Para el Manejo y Conservación de la Vida Silvestre (UMA) CIBAC, con registro DGVS-CR-IN 0952-D.F./07/UMA CIBAC.

\section{Alojamiento y obtención de espermatóforos}

Los ajolotes fueron alojados para su monitoreo durante un año de manera individual en contenedores de $60 \mathrm{~L}$, a una temperatura de $18^{\circ} \mathrm{C}$, con un fotoperiodo de $12 \mathrm{~h}$ luz y $12 \mathrm{~h}$ de obscuridad. Para estimular la liberación de espermatóforos, los machos se trasladaron a un contenedor de cristal con capacidad de $700 \mathrm{~L}$ de agua, el cual se acondicionó con suelo arenoso y plantas acuáticas; durante las horas de obscuridad, utilizando chiller de 0.25 HP (Ártica Resun CL-600), se redujo la temperatura del agua; de igual manera para todos los ejemplares de $18{ }^{\circ} \mathrm{C}$ a $14^{\circ} \mathrm{C}$, introduciendo 3 hembras por macho. Los espermatóforos fueron recuperados del fondo del contenedor, al inicio de las siguientes 12 horas de luz.

\section{Manejo de espermatóforos y obtención de espermatozoides}

Los espermatóforos liberados por cada ejemplar fueron recolectados a $5^{\circ} \mathrm{C}$ en $2 \mathrm{ml}$ de medio Simplified Amphibian Ringer (SAR), compuesto por $\mathrm{NaCl} 113 \mathrm{mM}, \mathrm{CaCl} 1 \mathrm{mM}, \mathrm{KCl}$ $2.0 \mathrm{mM}$ y NaHCO $3.6 \mathrm{mM}$, con $220 \mathrm{mOsmol} \mathrm{kg}^{-1}$. Los espermatozoides se obtuvieron colocando los espermatóforos de cada ejemplar en una caja Nunc ${ }^{\mathrm{MR}}$ de 4 pozos con 0.5 $\mathrm{ml}$ de medio SAR; en el primer pozo se lavaron para eliminar residuos de materia orgánica; en el segundo pozo se retiró el material gelatinoso (glucoproteínas) para obtener el capuchón que contiene los espermatozoides; en el tercer pozo se utilizó 0.5 $\mathrm{ml}$ de Hidróxido de Sodio $(\mathrm{NaOH})$ al $20 \%$, para reblandecer el capuchón por 10 min (Taku et al., 2004); en el cuarto pozo con $0.5 \mathrm{ml}$ de medio SAR, los espermatozoides se extrajeron por maceración del capuchón. Mediante aspiración se extrajeron los espermatozoides del sobrenadante, enseguida se filtraron con una malla de $30 \mu \mathrm{m}$ y se recuperó el total de espermatozoides de los espermatóforos de cada liberación en un 
tubo Eppendorf con $500 \mu \mathrm{l}$ de medio SAR a $5^{\circ} \mathrm{C}$, para realizar un pool espermático de cada liberación.

\section{Criopreservación espermática}

Cada pool espermático, conservado a $5^{\circ} \mathrm{C}$, se ajustó con $6 \%$ de Dimetilacetamida (DMA), enseguida se llenaron pajillas de $0.25 \mathrm{ml}$, para mantener en equilibrio por $10 \mathrm{~min}$ a $2^{\circ} \mathrm{C}$, luego se colocaron a $5 \mathrm{~cm}$ sobre vapor de nitrógeno a $-76{ }^{\circ} \mathrm{C}$ por 15 minutos y posteriormente se sumergieron en nitrógeno líquido a $-196^{\circ} \mathrm{C}$, para ser criopreservados por 30 días hasta su posterior descongelación a $15^{\circ} \mathrm{C}$ por 5 min (Atencio et al., 2013).

\section{Evaluación espermática básica}

El porcentaje de espermatozoides vivos, se determinó a través de un frotis que se realizó con una mezcla 1:5 espermatozoides con tinción de eosina-nigrosina, en la cual se contaron 100 espermatozoides bajo el microscopio a 40X; Se consideraron espermatozoides vivos, los no teñidos y muertos, los que presentaron tinción. La morfología espermática fue evaluada en la misma tinción, para determinar porcentajes de espermatozoides con alteraciones morfológicas en las regiones de la cabeza, cuello o flagelo (Tanisław et al., 2017).

\section{Distribución de carbohidratos membranales}

Con el uso de las lectinas de Triticum vulgaris aglutinina (WGA), con afinidad a residuos de N-Acetilglucosamina y de Arachis hypogaea (PNA) con afinidad a $\beta$-galactosa, aconjugadas a isotiocianato de fluoresceína (FICT), se pretendió determinar la presencia de los carbohidratos que se ha reportado son receptores para el reconocimiento entre los gametos (Herrera et al., 2017) y como partes estructurales del plasmalema espermático (Miller, 2015 ). En un volumen final de $40 \mu \mathrm{l}$ de medio SAR, con 5x106 espermatozoides, se adicionó $10 \mu \mathrm{l}$ de WGA-FICT o PNA-FITC a una concentración de $15 \mathrm{mg} / \mathrm{ml}$; se incubaron a $25^{\circ} \mathrm{C}$ por 30 minutos, estos espermatozoides cubriéndolos de la luz; enseguida se realizaron preparaciones sobre porta objetos, para observarlas al microscopio. Cada preparación se observó directamente en un microscopio de fluorescencia a $260 \mathrm{~nm}$ de excitación y >560 nm emisión, contabilizando 100 espermatozoides. Se determinó la presencia de los carbohidratos membranales, mediante patrones de fluorescencia y la proporción de espermatozoides con cada patrón determinado (Naofumi, 2015).

\section{Análisis estadístico}

Se determinó la frecuencia de espermatozoides vivos, con morfología normal y con los diferentes patrones de fluorescencia en las muestras frescas y descongeladas, las cuales fueron expresadas como proporción con su respectivo error estándar (EE). Las diferentes variables fueron comparadas entre los grupos de espermatozoides en fresco y 
descongeladas con una prueba de $\mathrm{Xi}^{2}$, con un una alfa de 0.05; utilizando el paquete estadístico de libre acceso Epilnfo 7.3

\section{RESULTADOS}

Se determinó diferente cantidad de eventos reproductivos y número de espermatóforos liberados por cada ejemplar; se obtuvieron un total de 61 espermatóforos. La frecuencia en la cantidad de espermatóforos liberados por ejemplar, fue la siguiente: 1:12, 2:10, 1:8, $1: 6,3: 4$ y 1:3. La concentración espermática tuvo un promedio de $2.6 \pm 0.6 \times 10^{4}$ espermatozoides/ $\mathrm{ml}$, con un rango entre $1.0 \pm 2.5$ a $4.0 \pm 3.0$. La concentración espermática en liberaciones con seis 0 más espermatóforos $\left(2.5 \times 10^{4}\right.$ espermatozoides $/ \mathrm{ml})$, fue mayor $(\mathrm{P}<0.05)$ a la concentración de espermatozoides $\left(2.5 \times 10^{4}\right.$ espermatozoides $\left./ \mathrm{ml}\right)$ en liberaciones con menos de seis espermatóforos.

\section{Parámetros de evaluación espermática}

Los porcentajes de espermatozoides vivos disminuyeron $(\mathrm{P}<0.05)$, aproximadamente 30 $\%$ en post descongelación; encontrando promedios de $89 \%$ en espermatozoides en fresco y del $58 \%$ post descongelación. Los porcentajes de espermatozoides vivos que se determinaron en liberaciones con diferente número de espermatóforos, mostró diferencias $(\mathrm{P}<0.05)$, encontrando un rango de $79 \%$ al $100 \%$ en espermatozoides en fresco y del $45 \%$ al $67 \%$, en espermatozoides descongelados (tabla 1 ).

La morfología espermática normal de igual manera mostró una reducción $(\mathrm{P}<0.05)$ de aproximadamente el $15 \%$, encontrando porcentajes del $98 \%$ en morfología normal de espermatozoides en fresco y del $83 \%$ post descongelación. Los porcentajes de espermatozoides con morfología normal que se determinaron en liberaciones con diferente número de espermatóforos, no mostró diferencias ( $P>0.05)$, encontrando un rango promedio de $95 \%$ al $100 \%$ en espermatozoides en fresco; sin embargo, determinados post descongelación fue diferente $(P<0.5)$, con porcentajes con rango promedio entre $78 \%$ al $90 \%$ (tabla1).

\section{Presencia y distribución de carbohidratos membranales}

Con el uso de la lectina WGA-FITC: En espermatozoides en fresco y post descongelación, la intensidad de la fluorescencia que emitió la lectina WGA-FITC sobre la membrana del espermatozoide, evidenció la presencia de residuos de $\mathrm{N}$-Acetil glucosamina presentes en la membrana espermática de $A$ mexicanum. Se determinaron dos patrones de fluorescencia denominados: Patrón A) Con fluorescencia intensa homogénea en la región del flagelo y cuello y con menor intensidad, pero evidente en la región de la cabeza (figura $1 \mathrm{~A}$ ); y patrón $\mathrm{B}$ ), con fluorescencia evidente homogénea en toda la estructura espermática (figura 1B). 
Tabla 1. Parámetros de evaluación espermática en fresco y post descongelación de $A$. mexicanum, en eyaculados de cada ejemplar evaluado

\begin{tabular}{|c|c|c|c|c|c|c|c|}
\hline \multirow[t]{2}{*}{ ID } & \multirow[t]{2}{*}{$\begin{array}{c}\text { Espermatóforos } \\
\quad \mathrm{n}=\end{array}$} & \multicolumn{3}{|c|}{$\begin{array}{l}\% \text { Espermatozoides } \\
\text { con Patrón } A \pm E E\end{array}$} & \multicolumn{3}{|c|}{$\begin{array}{l}\text { \% Espermatozoides } \\
\text { con Patrón B } \pm \mathrm{EE}\end{array}$} \\
\hline & & Fresco & Descongelado & $\begin{array}{c}X i^{2} \\
p\end{array}$ & Fresco & Descongelado & $\begin{array}{c}X i^{2} \\
p\end{array}$ \\
\hline A & 12 & $46 \pm 3$ & $51 \pm 7$ & $\begin{array}{c}0.3 \\
>0.05\end{array}$ & $54 \pm 3$ & $50 \pm 7$ & $\begin{array}{c}0.1 \\
>0.05\end{array}$ \\
\hline B & 10 & $44 \pm 3$ & $46 \pm 3$ & $\begin{array}{c}0.02 \\
>0.05\end{array}$ & $48 \pm 4$ & $53 \pm 3$ & $\begin{array}{c}0.3 \\
>0.05\end{array}$ \\
\hline C & 10 & $52 \pm 4$ & $56 \pm 6$ & $\begin{array}{c}0.2 \\
>0.05\end{array}$ & $47 \pm 4$ & $43 \pm 6$ & $\begin{array}{c}0.2 \\
>0.05\end{array}$ \\
\hline $\mathrm{D}$ & 8 & $56 \pm 5$ & $55 \pm 8$ & $\begin{array}{c}0.01 \\
>0.05\end{array}$ & $46 \pm 5$ & $46 \pm 8$ & $\begin{array}{l}1.0 \\
>0.05\end{array}$ \\
\hline $\mathrm{E}$ & 6 & $52 \pm 3.5$ & $57 \pm 9$ & $\begin{aligned} & 0.3 \\
&> 0.05 \\
&\end{aligned}$ & $47 \pm 3$ & $43 \pm 9$ & $\begin{array}{c}0.2 \\
>0.05 \\
\end{array}$ \\
\hline $\mathrm{F}$ & 4 & $49 \pm 7.7$ & $57 \pm 7$ & $\begin{array}{c}0.9 \\
>0.05\end{array}$ & $61 \pm 4$ & $42 \pm 7$ & $\begin{array}{r}\quad 6.5 \\
<0.05\end{array}$ \\
\hline $\mathrm{G}$ & 4 & $41 \pm 4.2$ & $55 \pm 6$ & $\begin{array}{c}3.9 \\
<0.05\end{array}$ & $59 \pm 4^{a}$ & $45 \pm 6$ & $\begin{array}{c}3.9 \\
<0.05\end{array}$ \\
\hline $\mathrm{H}$ & 4 & $45 \pm 7.3$ & $56 \pm 6$ & $\begin{array}{c}2 \\
<0.05\end{array}$ & $55 \pm 7$ & $44 \pm 6$ & $\begin{array}{c}2.0 \\
>0.05\end{array}$ \\
\hline$T$ & 3 & $45 \pm 2.8$ & $55 \pm 5$ & $\begin{array}{c}1.6 \\
<0.05\end{array}$ & $65 \pm 3$ & $45 \pm 5$ & $\begin{array}{c}7.3 \\
<0.05\end{array}$ \\
\hline
\end{tabular}

Literal diferente en súper índice $(a, b, c)$, indica diferencia $(P<0.05)$ al comparar la misma variable entre columnas (Fresco VS Descongelado). Número diferente en súper índice ${ }^{(1,2,3)}$, indica diferencia $(\mathrm{P}<0.05)$ al comparar los promedios en la misma columna

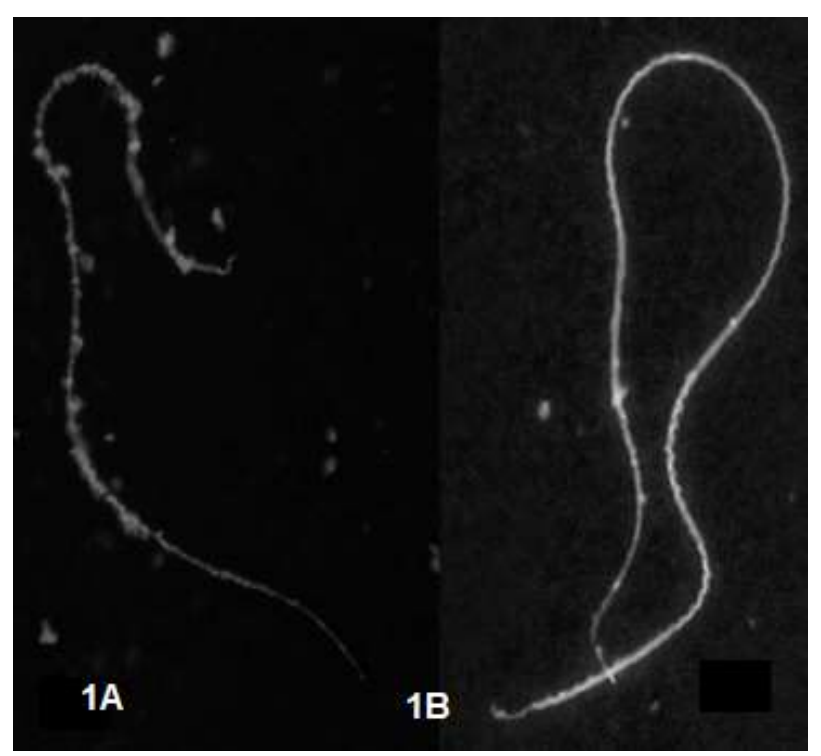

Figura. 1. Patrones de fluorescencia de espermatozoide con la lectina WGA-FITC. 1A). Se observa mayor intensidad de fluorescencia en las regiones del flagelo y cuellos y con menor intensidad en la cabeza. 1B se observa con intensidad homogénea en toda la estructura espermática 
La proporción de patrones determinados con WGA-FITC (tabla 2), mostraron que:

El porcentaje de espermatozoides con patrón A, procedentes de liberaciones de doce a seis espermatóforos, fueron similares $(P>0.05)$ en fresco y post descongelación; en comparación con los porcentajes de espermatozoides procedentes de liberaciones entre ocho y tres espermatóforos, en los que se incrementó $(P<0.05)$, en espermatozoides descongelados. Al comparar los porcentajes de espermatozoides con patrón A, obtenidos en espermatozoides de cada ejemplar en fresco, se determinó en promedio $48 \%$, con rango entre $44 \%$ a 56\%; sin encontrar diferencia $(P<0.05)$ entre éstos, en espermatozoides descongelados; se determinó un promedio de $54 \%$ con rango entre $46 \%$ a $57 \%$, encontrando porcentajes mayores ( $P>0.05)$, en espermatozoides con el patrón $A$, procedentes de liberaciones con 3 y 4 espermatóforos.

Los porcentajes de espermatozoides con patrón $B$, procedentes de liberaciones de doce a seis espermatóforos fueron similares $(P>0.05)$ en fresco y post descongelación; los porcentajes de espermatozoides procedentes de liberaciones con cuatro y tres espermatóforos, fueron mayores $(\mathrm{P}<0.05)$ en fresco, comparados con los descongelados. En fresco se determinó en promedio 53\%, con rango entre 46 a $65 \%$, sin encontrar diferencia $(\mathrm{P}<0.05)$ entre éstos; en espermatozoides descongelados, se determinó un promedio de $46 \%$ con rango entre $42 \%$ a $53 \%$, encontrando porcentajes mayores $(P>0.05)$ en espermatozoides procedentes de liberaciones con 10 y 12 espermatóforos. Al realizar la comparación general total (Pool) de los porcentajes, fue evidente una diferencia y de manera inversa el por porcentajes totales determinados, los cuales fueron para espermatozoides con el Patrón A: $X i^{2}$ de $7.21 p<0.05$, en semen fresco con $47.7 \%$ y de $54.3 \%$ para semen descongelado; con respecto a espermatozoides con el Patrón B: se determinó una $X i^{2}$ de $10.8 p<0.05$, en semen fresco con $53.5 \%$ y de $45.6 \%$ en semen descongelado.

Con el uso de la lectina PNA-FITC: en espermatozoides en fresco y post descongelación, la intensidad de la fluorescencia que emitió la lectina PNA-FITC sobre la membrana del espermatozoide, lo que evidenció la presencia de residuos glicosídicos de $\beta$-galactosa, presentes en la membrana del espermatozoide de $A$ mexicanum. Se determinaron dos patrones de fluorescencia: $C$ ) Con fluorescencia intensa y homogénea a lo largo de toda la estructura espermática (figura 2A); patrón D) Con fluorescencia tenue homogénea en toda la estructura espermática (figura 2B).

La proporción de patrones determinados con PNA-FITC, (tabla 3), mostró que:

Con los patrones $\boldsymbol{C} \boldsymbol{y} \boldsymbol{D}$, los porcentajes de espermatozoides procedentes de liberaciones entre 6 a 12 espermatóforos fueron similares $(P>0.05)$ en fresco y post descongelación, en comparación con los porcentajes de espermatozoides procedentes de liberaciones con tres y cuatro espermatóforos, que se incrementaron $(P<0.05)$, en espermatozoides descongelados. 
Tabla 2. Porcentajes de espermatozoides en fresco y post descongelación, con dos patrones de fluorescencia A y B determinados con el uso de la lectina WGA-FITC

\begin{tabular}{|c|c|c|c|c|c|c|c|}
\hline \multirow[t]{2}{*}{ ID } & \multirow[t]{2}{*}{$\begin{array}{c}\text { Espermatóforos } \\
\quad \mathrm{n}=\end{array}$} & \multicolumn{3}{|c|}{$\begin{array}{c}\text { \% Espermatozoides } \\
\text { con Patrón } \mathrm{A} \pm \mathrm{EE}\end{array}$} & \multicolumn{3}{|c|}{$\begin{array}{c}\text { \% Espermatozoides } \\
\text { con Patrón } \mathrm{B} \pm \mathrm{EE}\end{array}$} \\
\hline & & Fresco & Descongelado & $\begin{array}{c}X i^{2} \\
p\end{array}$ & Fresco & Descongelado & $\begin{array}{c}X i^{2} \\
p\end{array}$ \\
\hline A & 12 & $46 \pm 3$ & $51 \pm 7$ & $\begin{array}{c}0.3 \\
>0.05\end{array}$ & $54 \pm 3$ & $50 \pm 7$ & $\begin{array}{c}0.1 \\
>0.05\end{array}$ \\
\hline B & 10 & $44 \pm 3$ & $46 \pm 3$ & $\begin{array}{c}0.02 \\
>0.05\end{array}$ & $48 \pm 4$ & $53 \pm 3$ & $\begin{array}{c}0.3 \\
>0.05\end{array}$ \\
\hline $\mathrm{C}$ & 10 & $52 \pm 4$ & $56 \pm 6$ & $\begin{array}{c}0.2 \\
>0.05\end{array}$ & $47 \pm 4$ & $43 \pm 6$ & $\begin{array}{c}0.2 \\
>0.05\end{array}$ \\
\hline $\bar{D}$ & 8 & $56 \pm 5$ & $55 \pm 8$ & $\begin{array}{c}0.01 \\
>0.05\end{array}$ & $46 \pm 5$ & $46 \pm 8$ & $\begin{array}{c}1.0 \\
>0.05\end{array}$ \\
\hline $\mathrm{E}$ & 6 & $52 \pm 3.5$ & $57 \pm 9$ & $\begin{array}{c}0.3 \\
>0.05\end{array}$ & $47 \pm 3$ & $43 \pm 9$ & $\begin{array}{c}0.2 \\
>0.05\end{array}$ \\
\hline $\mathrm{F}$ & 4 & $49 \pm 7.7$ & $57 \pm 7$ & $\begin{array}{c}0.9 \\
>0.05\end{array}$ & $61 \pm 4$ & $42 \pm 7$ & $\begin{array}{c}6.5 \\
<0.05\end{array}$ \\
\hline G & 4 & $41 \pm 4.2$ & $55 \pm 6$ & $\begin{array}{c}3.9 \\
<0.05\end{array}$ & $59 \pm 4^{a}$ & $45 \pm 6$ & $\begin{array}{c}3.9 \\
<0.05\end{array}$ \\
\hline $\mathrm{H}$ & 4 & $45 \pm 7.3$ & $56 \pm 6$ & $\begin{array}{c}2 \\
<0.05\end{array}$ & $55 \pm 7$ & $44 \pm 6$ & $\begin{array}{c}2.0 \\
>0.05\end{array}$ \\
\hline$T$ & 3 & $45 \pm 2.8$ & $55 \pm 5$ & $\begin{array}{c}1.6 \\
<0.05\end{array}$ & $65 \pm 3$ & $45 \pm 5$ & $\begin{array}{c}7.3 \\
<0.05\end{array}$ \\
\hline
\end{tabular}

Literal diferente en súper índice $(a, b, c)$, indica diferencia $(\mathrm{P}<0.05)$ al comparar la misma variable entre columnas (Fresco vs Descongelado).

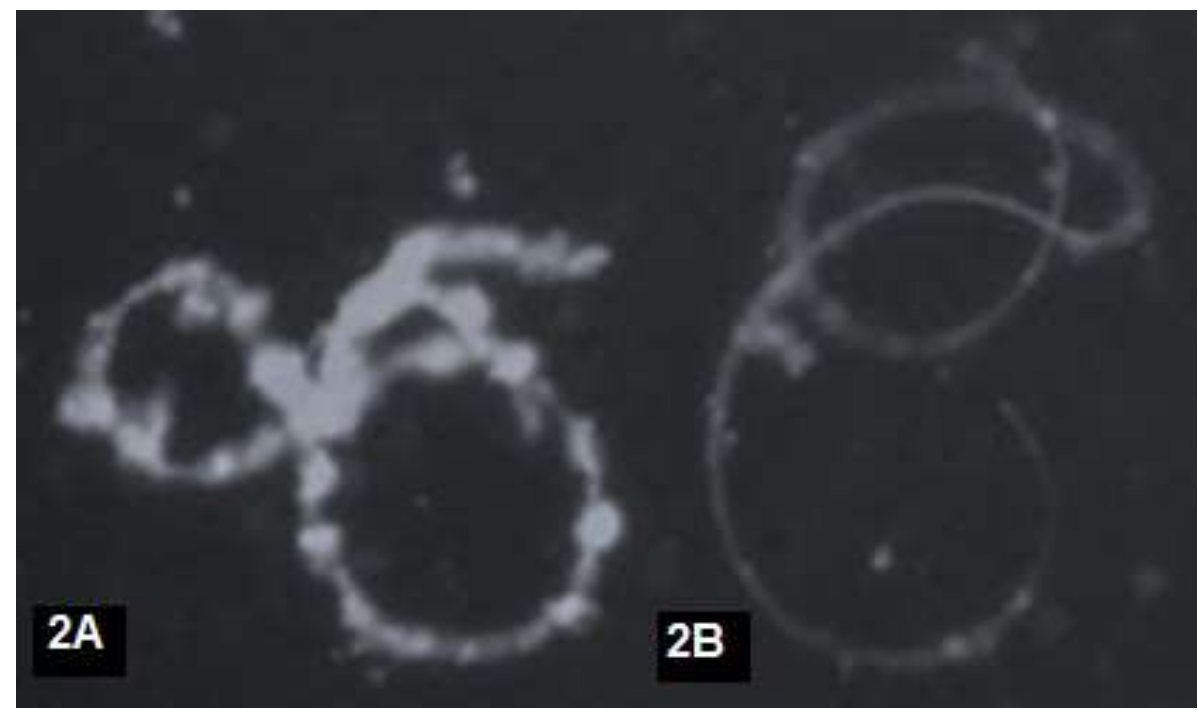

Figura 2. Patrones de fluorescencia obtenidos con lectina PNA-FITC: A) Con fluorescencia intensa homogénea, B) Con fluorescencia tenue homogénea 
Los porcentajes de espertmatozoides con patrón $\mathrm{C}$ y $\mathrm{D}$, determinados con la lectina PNAFITC, únicamente mostraron diferencia $(P<0.05)$ post descongelación, cuando los espermatoziodes provinieron de liberaciones con ocho espermatóforos, observando mayor porcentaje en espermatozoides con patrón $\mathrm{C}$ post descongelación y de manera inversa, en espermatozoides con el patrón D. El porcentaje fue mayor en semen fresco, en los porcentajes de espermatozoides provenientes de liberaciones con doce, diez, seis, cuatro o tres espermatóforos, Al comparar los porcentajes de espermatozoides con el Patrón $C$ de cada ejemplar, en fresco con un promedio de $54 \%$, con rango entre $49 \%$ a $58 \%$ y post descongelación con promedio de $60 \%$ y con rango entre $47 \%$ a $69 \%$, no se encontraron diferencias $(\mathrm{P}<0.05)$.

Al comparar los porcentajes de espermatozoides con patrón $\boldsymbol{D}$ de cada ejemplar, en fresco con un promedio de $48 \%$, con rango entre $42 \%$ a $55 \%$ no se observaron porcentajes diferentes $(P<0.05)$. En espermatozoides descongelados se encontró en promedio de $42 \%$ con rango entre $24 \%$ a $53 \%$, tampoco se observaron porcentajes diferencias $(\mathrm{P}<0.05)$.

Tabla 3. Porcentajes de espermatozoides en fresco y post descongelación, con dos patrones de fluorescencia C y D determinados con el uso de la lectina PNA-FITC

\begin{tabular}{|c|c|c|c|c|c|c|c|}
\hline \multirow[t]{2}{*}{ ID } & \multirow[t]{2}{*}{$\begin{array}{l}\text { Espermatóforos } \\
\qquad \mathrm{n}=\end{array}$} & \multicolumn{2}{|c|}{$\begin{array}{c}\% \pm \mathrm{EE} \\
\text { Espermatozoides } \\
\text { con Patrón C }\end{array}$} & \multicolumn{4}{|c|}{$\begin{array}{c}\% \pm \mathrm{EE} \\
\text { Espermatozoides } \\
\text { con Patrón D }\end{array}$} \\
\hline & & Fresco & Descongelado & $\begin{array}{c}X i^{2} \\
p \\
\end{array}$ & Fresco & Descongelado & $\begin{array}{c}X{ }^{2} \\
p\end{array}$ \\
\hline A & 12 & $51 \pm 4$ & $47 \pm 5$ & $\begin{array}{c}0.2 \\
>0.05\end{array}$ & $49 \pm 4$ & $53 \pm 5$ & $\begin{array}{c}0.2 \\
>0.05\end{array}$ \\
\hline B & 10 & $51 \pm 5$ & $49 \pm 5$ & $\begin{array}{c}0.02 \\
>0.05\end{array}$ & $49 \pm 5$ & $50 \pm 5$ & $\begin{array}{c}0.01 \\
>0.05\end{array}$ \\
\hline C & 10 & $53 \pm 5$ & $52 \pm 6$ & $\begin{array}{c}0.01 \\
>0.05\end{array}$ & $48 \pm 7$ & $47 \pm 6$ & $\begin{array}{c}0.01 \\
>0.05\end{array}$ \\
\hline D & 8 & $49 \pm 4$ & $66 \pm 3$ & $\begin{array}{c}5.23 \\
<0.05\end{array}$ & $51 \pm 5$ & $24 \pm 3$ & $\begin{array}{c}14.4 \\
<0.05\end{array}$ \\
\hline$E$ & 6 & $57 \pm 4$ & $60 \pm 7$ & $\begin{array}{c}0.08 \\
>0.05\end{array}$ & $47 \pm 7$ & $40 \pm 11$ & $\begin{array}{c}0.7 \\
>0.05\end{array}$ \\
\hline$F$ & 4 & $55 \pm 7$ & $62 \pm 4$ & $\begin{array}{c}0.07 \\
>0.05\end{array}$ & $45 \pm 10$ & $37 \pm 4$ & $\begin{array}{c}1.01 \\
>0.05\end{array}$ \\
\hline G & 4 & $55 \pm 4$ & $68 \pm 6$ & $\begin{aligned} & 0.7 \\
> & 0.05\end{aligned}$ & $55 \pm 6$ & $44 \pm 7$ & $\begin{aligned} & 2.0 \\
> & 0.05\end{aligned}$ \\
\hline $\mathrm{H}$ & 4 & $57 \pm 3$ & $69 \pm 4$ & $\begin{aligned} & 2.6 \\
> & 0.05\end{aligned}$ & $52 \pm 6$ & $42 \pm 6$ & $\begin{array}{c}1.62 \\
>0.05\end{array}$ \\
\hline I & 3 & $58 \pm 5$ & $67 \pm 5$ & $\begin{array}{l}1.36 \\
>0.05\end{array}$ & $42 \pm 8$ & $43 \pm 9$ & $\begin{array}{c}0.01 \\
>0.05\end{array}$ \\
\hline
\end{tabular}

Literales diferentes en súper índice ${ }^{(a, b, c)}$, indica diferencia $(\mathrm{P}<0.05)$ al comparar la misma variable entre columnas (Fresco VS Descongelado). 


\section{DISCUSIÓN}

En cuanto a la concentración espermática, los datos mostraron que existen diferencias entre espermatóforos de un mismo ejemplar y entre ejemplares. Estos resultados son similares a los publicados por Doyle et al. (2011), en el número de espermatozoides entre los espermatóforos liberados de ejemplares de A maculatum. La cantidad y el tamaño de los espermatóforos que pueden ser liberados varía ampliamente, relacionándose con la fisiología y adaptación reproductiva de cada especie (Browne et al., 2019); así como de tres características físicas: tamaño corporal, tamaño de los testículos o la edad (Uribe y Mejía-Roa, 2014), lo cual también fue observado en nuestro estudio. La viabilidad espermática observada en fresco fue de 80 a $98 \%$, siendo este el primer trabajo que registra el porcentaje de espermatozoides vivos extraídos de los espermatóforos. Estos resultados se contraponen a los reportados por Mansour et al. (2011), quienes obtuvieron espermatozoides por medio de masaje cloacal, reportando un $100 \%$ de espermatozoides vivos en todas las muestras analizadas.

Por otra parte, un estudio realizado por Chester (2013), menciona que obtuvo de 64 a 86 $\%$ de viabilidad espermática después de llevar a cabo la criopreservación de espermatóforos. Estos datos difieren de lo obtenido en este trabajo, donde la viabilidad obtenida en espermatozoides descongelados estuvo en un promedio de 45 a $68 \%$, por lo que este resultado nos indica que la membrana del capuchón puede funcionar como una barrera, la cual protege a los espermatozoides de los cambios bruscos de la congelación (Chester, 2013; Hall et al., 2016).

La utilización de lectinas WGA-FITC y PNA-FITC mostró ser una alternativa para identificar la presencia y distribución de los residuos glucosídicos B-galactosa y Acetilglucosamina. Estos resultados concuerdan con el trabajo realizado por Sáez et al., (2004), en donde se determinó que en el momento de la espermatogénesis diferentes carbohidratos entre ellos B-galactosa y acetilglucosamina se presentan en la membrana de las células que se encuentran en diferente etapa de desarrollo durante la espermiogénesis.

La presencia y distribución de los residuos glucosídicos, indica diferencias a lo largo de toda la membrana, lo cual se determinó con cada lectina, que cada una identificó al menos dos patrones de fluorescencia diferentes; los cuales pueden estar asociados a diferentes estados metabólicos de los espermatozoides que les permitan o no el reconocimiento entre gametos.

La presencia y distribución de los residuos glucosídicos permite caracterizar la membrana de los espermatozoides que se encuentren en diferente estado metabólico, asociados a la capacitación y reacción acrosomal, y por lo cual su capacidad fertilizante, 
(Browne et al., 2015). Lo anterior puede ser de utilidad en protocolos de reproducción asistida que involucren el manejo in vitro de los espermatozoides.

Utilizando la reproducción asistida en cautiverio, se contribuye a la conservación de la especie; además de disminuir la extracción de animales de su medio y venta ilegal (Jimenez et al., 2017); los cuales pueden tener un aprovechamiento sustentable destinando ejemplares a la conservación, investigación biomédica y conservación en colecciones públicas y privadas (Prieto et al., 2014), que es en estas últimas, en las cuales es donde se han reproducido los ejemplares fuera de su hábitat natural; sin embargo, la reproducción de anfibios y su cría en cautiverio es relativamente mínimo (Ananjeva et al., 2015).

\section{CONCLUSIÓN}

El protocolo de criopreservación utilizado demostró ser eficiente, manteniendo parámetros de viabilidad e integridad membranal, a pesar de encontrar diferencias espermáticas asociadas al número de espermatóforos presentes en cada liberación, por lo cual este estudio aporta herramientas y conocimientos para la reproducción asistida en cautiverio del Ambystoma mexicanum.

\section{LITERATURA CITADA}

ANANJEVA NB, Uteshev VK, Orlov NL, Gakhova EN. 2015. Strategies for conservation of endangered amphibian and reptile species. Biological Bulletin. 42:432-439. https://link.springer.com/article/10.1134/S1062359015050027

ATENCIO V, Pérez E, Espinosa J, Pardo S. 2013. Evaluación de dimetilacetamida como crioprotector para la crioconservación de semen de Bocachico prochilodus magdalenae. Archivos de Medicina Veterinaria. 45(2):151-158.

http://dx.doi.org/10.4067/S0301-732X2013000200006

BROWNE R, Chester R. Figiel Jr. 2011. Amphibian conservation and cryopreservation of sperm, cells, and tissues. In Cryopreservation in Aquatic Species. 8(3):345-365. http://www.herpconbio.org/Volume_8/Issue_3/Figiel_2013.pdf

BROWNE RK, Kaurova SA, Uteshev VK, Shishova NV, McGinnity D, Figiel CR, Mansour N, Agnew D, Wu M, Gakhova EN, Dzyuba B, Cosson J. 2015. Sperm motility of externally fertilizing fish ans amphibians. Theriogenology. 83(1):1-13. https://doi.org/10.1016/j.theriogenology.2014.09.018 
BROWNE RK, Silla AJ, Upton R, Della-Togna G, Marcec-Greaves R, Shishova NV, Uteshev VK, Proaño B, Pérez OD, Mansour N, Kaurova SA, Gakhova EN, Cosson J, Dyzuba B, Kramarova LI, McGinnity D, Gonzalez M, Clulow J, Clulow S. 2019. Sperm collection and storage for the sustainable management of amphibian biodiversity. Theriogenology. 5:133:187-200. https://doi.org/10.1016/j.theriogenology.2019.03.035. PMID: 31155034.

CATENAZZI A. 2015. State of the world's amphibians. Annual Review of Environment and Resources. 40:91-119. https://doi.org/10.1146/annurev-environ-102014-021358

CLULOW J, Trudeau VL, Kouba AJ. 2014. Amphibian declines in the twenty-first century: Why we need assisted reproductive technologies. In Reproductive Sciences in Animal Conservation, W.V. Holt, J.L. Brown, and P. Comizzoli, eds. (New York, NY: Springer New York), pp. 275-316. https://doi.org/10.1007/978-1-4939-0820-2_12

COMIZZOLI P, Songsasen N, Hagedorn M, Wildt DE. 2012. Comparative cryobiological traits and requirements for gametes and gonadal tissues collected from wildlife species. Theriogenology. 78(8):1666-1681. https://doi.org/10.1016/j.theriogenology.2012.04.008.

CHESTER RF. 2013. Cryopreservation of sperm from the axolotl Ambystoma mexicanum: implications for conservation. Herpetological Conservation and Biology. 8(3):748-755. http://www.herpconbio.org/Volume_8/lssue_3/Figiel_2013.pdf

DOYLE JM, McCormick CR, De Woody JA. 2011. The quantification of spermatozoa by real-time quantitative PCR, spectrophotometry, and spermatophore cap size: Technical advances. Molecular Ecology Resources. 11(1):101-106.

https://doi.org/10.1111/j.1755-0998.2010.02892.x.

EPI. Info R7. 2020. paquete estadístico de libre acceso Epi. Info 7R.

https://www.cdc.gov/epiinfo/esp/es_pc.html

HERRERA JA, Calderón G, Cruz C, Ávila MA, Quintero GE, Fierro RC. 2017. Changes in the membrane carbohydrates from sperm cryopreserved with dimethylsulfoxide or polyvinylpyrrolidone of red-tailed hawk (Buteo jamaicencis). Cryo Letters. 38(4):257-262. PMID: 29734426. https://pubmed.ncbi.nlm.nih.gov/29734426/

HALL KW, Eisthen HL, Williams BL. 2016. Proteinaceous pheromone homologs identified from the cloacal gland transcriptome of a male axolotl, Ambystoma mexicanum. PLOS ONE 11, e0146851. https://doi.org/10.1371/journal.pone.0146851

JIMÉNEZ JO, Aviña CR, Ramírez AE, Lucero FG, Andreu CG. 2017. Conservación exsitu de poblaciones en riesgo de ajolotes (Ambystoma spp.) del estado de puebla, Mexico. Revista Latinoamericana el Ambiente y las Ciencias. 8(18):1-10.

http://cmas.siu.buap.mx/portal_pprd/work/sites/rlac/resources/LocalContent/90/1/8(18)1.pdf 
KHATTAK S, Murawala P, Andreas H, Kappert V, Schuez M, Sandoval-Guzmán T, Crawford K, Tanaka EM. 2014. Optimized axolotl (Ambystoma mexicanum) husbandry, breeding, metamorphosis, transgenesis and tamoxifen-promedioted recombination. Nature Protocols. 9(3):529-540. https://doi.org/10.1038/nprot.2014.040

NOM-059-SEMARNAT-2010, Norma Oficial Mexicana. Protección ambiental-Especies nativas de México de flora y fauna silvestres-Categorías de riesgo y especificaciones para su inclusión, exclusión o cambio-Lista de especies en riesgo.

https://www.profepa.gob.mx/innovaportal/file/435/1/NOM_059_SEMARNAT_2010.pdf

MANSOUR N, Lahnsteiner F, Patzner RA. 2011. Collection of gametes from live axolotl, Ambystoma mexicanum, and standardization of in vitro fertilization. Theriogenology. 75(2): 354-361. https://doi.org/10.1016/j.theriogenology.2010.09.006

MENDOZA VT. 2012. Importancia ecológica y cultural de una especie endémica de ajolote (Ambystoma dumerilii) del lago de Patzcuaro Michoacan. Etnobiología. 10(2):4049. https://revistaetnobiologia.mx/index.php/etno/article/view/212/213

Miller DJ. 2015. Regulation of Sperm Function by Oviduct Fluid and the Epithelium: Insight into the Role of Glycans. Reproduction in Domestic Animals. 50 Suppl (2):31-39.

PMID: 26174917 DOI: 10.1111/rda.12570

NAOFUMI M. 2015. Protein-carbohydrate interaction between sperm and the egg-coating envelope and its regulation by dicalcin, a Xenopus laevis, zona pellucida proteinassociated protein. Molecules. 20(5):9468-9486.

https://doi.org/10.3390/molecules20059468

PRIETO MT, Sanchez-Calabuig MJ, Hildebrandt TB, Santiago-Moreno J. Saragusty J. 2014. Sperm cryopreservation in wild animals. European Journal of Wildlife Research. 60: 851-864. https://link.springer.com/article/10.1007\%2Fs10344-014-0858-4

SÁEZ FJ, Madrid JF, Cardoso S, Gómez L, Hernández F. 2004. Glycoconjugates of the urodele amphibian testis shown by lectin cytochemical methods. Microscopy Research and Technique. 64(1):63-76. https://doi.org/10.1002/jemt.20059

SHISHOVA NR, Uteshev VK, Kaurova SA, Browne RK, Gakhova EN. 2011. Cryopreservation of hormonally induced sperm for the conservation of threatened amphibians with rana temporaria as a model research species. Theriogenology. 75(2):220-232. https://doi.org/10.1016/j.theriogenology.2010.08.008

SILLA AJ, y Byrne PG. 2019. The Role of Reproductive Technologies in Amphibian Conservation Breeding Programs. Annual Review of Animal Biosciences. 7(1):499-519. https://doi.org/10.1146/annurev-animal-020518-115056 
SUNNY A, Monroy-Vilchis O, Fajardo V, Aguilera-Reyes U. 2014. Genetic diversity and structure of an endemic and critically endangered stream river salamander (Caudata: Ambystoma leorae) in México. Conservation Genetics. 15:49-59.

https://link.springer.com/article/10.1007/s10592-013-0520-9

TANISŁAW K, Anna W, Magdalena K, Krzysztof G. 2017. Application of two staining methods for sperm morphometric evaluation in domestic pigs. Journal of Veterinary Research. 61(3):345-349. https://doi.org/10.1515/jvetres-2017-0045

PELÁEZ J, Bongalhardo DC, Long JA. 2011. Characterizing the glycocalyx of poultry spermatozoa: III. Semen cryopreservation methods alter the carbohydrate component of rooster sperm membrane glycoconjugates. Poultry Science. 90(2):435-43. https://doi.org/10.3382/ps.2010-00998.

TAKU S, Masakazu A, Seiji G. 2004. A new method to extract sperm from spermatophores of the male spiny king crab $\mathrm{P}$ aralithodes brevipes (Anomura: lithodidae). Crustacean Research. (33):10-14. https://www.jstage.jst.go.jp/article/crustacea/33/0/33_KJ00004479508/_pdf

IUCN the Red List of Threatened Species. 2020. Disponible en línea en: https://www.iucnredlist.org/search?query=AMBYSTOMA\%20MEXICANUM\&searchType $=$ species

URIBE MC, Mejía-Roa V. 2014. Testicular structure and germ cells morphology in salamanders. Spermatogenesis. $\quad 4, \quad$ e988090. https://doi.org/10.4161/21565562.2014.988090

TIETJE M, Rödel M. 2018. Evaluating the predicted extinction risk of living amphibian species with the fossil record. Ecology Letters. 21(8):1135-1142.

https://onlinelibrary.wiley.com/doi/full/10.1111/ele.13080 